\title{
Ultrasound-Guided Totally Implantable Venous Access: Comparison between Arm and Chest Ports in Solid Organ Cancer Patients
}

\author{
Dong Hyun Lim ${ }^{1}$, Jae Do Yang ${ }^{1}$, Byeoung Hoon Chung ${ }^{1}$, Hee Chul Yu${ }^{1}$, Mi Rin Lee ${ }^{1}$, Hong Pil Hwang ${ }^{1,2,3}$ \\ ${ }^{1}$ Department of Surgery, Jeonbuk National University Medical School and Hospital, ${ }^{2}$ Biomedical Research Institute, Jeonbuk National \\ University Hospital, ${ }^{3}$ Research Institute of Clinical Medicine, Jeonbuk National University, Jeonju, Korea
}

Received August 4, 2021

Revised August 18, 2021

Accepted October 19, 2021

\section{Correspondence to:}

Hong Pil Hwang

Department of Surgery, Jeonbuk

National University Medical School

and Hospital, 20 Geonji-ro

Deokjin-gu, Jeonju 54907, Korea

Tel: + 82-63-250-1570

Fax: +82-63-271-6197

E-mail: h2p@jbnu.ac.kr
Purpose: The purpose of this study was to evaluate the safety, technical feasibility, and complications of ultrasound-guided placement of totally implantable venous access ports (TIVAPs) in the arm compared to the chest for patients with cancer in a single center. Methods: We retrospectively identified 371 patients who underwent TIVAP implantation in the upper arm or chest between July 2018 and June 2019. Implantation via the upper arm (arm port) or the jugular vein (chest port) was performed under sonographic and fluoroscopic guidance after administering local anesthesia. Medical records were reviewed to determine technical success, complications, and the causes of port removal were analyzed.

Results: In total, 371 devices were implanted, 252 in the upper arm $(\mathrm{n}=252)$ and 119 in the upper chest wall $(n=119)$. The technical success rate was $100 \%$. There were fewer complications observed in the arm port group compared to the chest port group (13 vs 23 patients; $5.2 \%$ vs $19.3 \%)$, which was statistically significant $(P=0.002)$. The chest port group developed more complications per 1000 catheter days and had a higher total complication rate than the arm port group $(\mathrm{P}<0.001)$. Local infection with abscess and wound dehiscence were the most common cause of port removal. Multivariate analysis showed that the implantable port in the arm involved a less complicated procedure $(\mathrm{P}=0.002)$.

Conclusion: Implantation of TIVAPs in the upper arm is a safe and feasible procedure with a low rate of complications. TIVAPs in the upper arm may be a good alternative to TIVAPs in the chest.

Keywords: Neoplasms, Drug therapy, Vascular access devices, Arm

\section{INTRODUCTION}

Totally implantable venous access ports (TIVAPs) are widely used vascular access methods for total parenteral nutrition, fluids, blood products, administration of medical agents, chemotherapy, and hemodynamic monitoring.(1) TIVAPs were introduced in the early 1980s and are currently the most common method for chemotherapy because of their various advantages in long-term administration.(2) Most oncologic guidelines recommend implantable port placement if chemotherapy is required for more than 3 months. (2-4)

Traditionally, TIVAPs were inserted via the internal jugular vein and the injection port was mounted on the anterior chest wall. However, this method cannot be applied to all patients, specifically those with malignancies in the neck or a tracheostomy.(5) In addition, many patients are 
uncomfortable about having a port inserted in the chest wall, especially breast cancer patients with large lesions. (5, 6) Recently, there has been an increase in the use of implantable ports in the arm, especially in breast cancer patients, because of the simplicity of the procedure and cosmetic superiority. $(2,6)$ However, few clinical trials have investigated the safety and stability of the procedure in various types of cancers. Other studies have reported that arm ports have a higher rate of complications than chest ports, especially with regard to catheter-induced thrombosis.(7) Against this background, we aimed to determine the differences in the safety and clinical usefulness of ultrasound-guided placement of implantable ports in the arm compared to the chest when used in solid organ cancer patients.

\section{METHODS}

\section{Study protocol}

This is a retrospective analysis comparing the clinical safety and usefulness of TIVAPs by evaluating the medical records of patients who underwent port insertion in the chest or arm at our institution from January 2018 to December 2019. The patients included in this study had cancer involving the breast, stomach, colon, rectum, and pancreas. This study was approved by the Institutional Review Board of our institution (CUH 2020-02-028-003).

\section{Surgical procedure in the arm}

We used a port system consisting of a titanium reservoir with a silicone rubber septum for needle insertion (PowerPort, Bard Access Systems, Salt Lake City, Utah, USA). All patients underwent preoperative evaluation of complete blood count, coagulation profile, and liver function tests. Preoperative ultrasound was performed by the operator to evaluate for optimal vein selection. The patient was placed in a supine position. After the arm was abducted, asepsis and antisepsis of the skin was performed by cleansing the area with 70\% isopropyl alcohol and 4\% chlorhexidine solution. After placing a tourniquet on the prox- imal upper arm, the location and diameter of the vein to be accessed and surrounding structures were determined by ultrasound. When using the arm veins, the most appropriate vein was selected by evaluating the diameter of each vein $(>3.0 \mathrm{~mm})$ and assessing for the presence of intravenous thrombi and continuity. Following local anesthesia around the needling site, the procedure was initiated by inserting a puncture needle, microwire, and catheter via ultrasound-guided Seldinger technique into the target vein of the arm. After placing the guidewire and introducer sheath, the catheter tip was positioned between the superior vena cava and the right atrium under fluoroscopic guidance. Subsequently, a pocket for the port device was created on the middle to lateral side of the upper arm. It is important to note that the pocket was created considering suitable depth, without being too thin or difficult to access. After connecting to the catheter, the port was inserted into the subcutaneous pocket. After careful hemostasis, the subcutaneous fat layer was sutured by aligning the skin margins, and the skin was approximated to coaptation using glue (Fig. 1A).

\section{Surgical procedure in chest}

The preoperative preparation was the same as that in the arm. Under ultrasound guidance, the right or left internal jugular vein was punctured with a 20 -gauge needle, and a
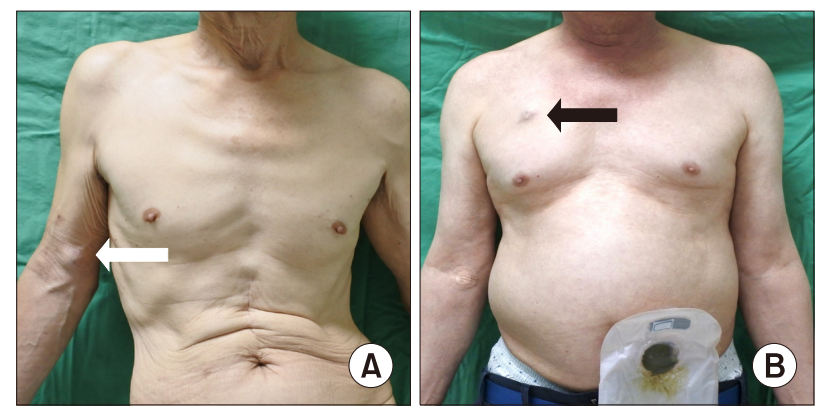

Fig. 1. Patients who underwent arm port and chest port. (A) A 63-year-old male patient with right colorectal cancer underwent arm port procedure (white arrow) on the right upper arm for adjuvant chemotherapy after right hemicolectomy. (B) A 68-year-old male patient underwent conventional port procedure (black arrow) on the right upper chest wall for adjuvant chemotherapy after Miles operation and for ultra-lower rectal cancer. 
catheter was inserted along the guidewire. Subsequently, a pocket was made in the subcutaneous fat layer to insert the port 3-5 $\mathrm{cm}$ below the middle of the clavicle at the upper part of the chest. The subsequent procedure was the same as that for the arm (Fig. 1B).

\section{Statistical analysis}

Descriptive variables were presented as mean \pm standard deviation, and categorical variables were described as counts and percentages. We used the student's t-test and chi-square test to compare parametric and nonparametric variables between groups. A logistic regression model was applied to assess the risk factors for catheter-related infections. Factors with a $\mathrm{P}<0.20$, on univariate analysis, were selected for multivariate analysis. The Kaplan-Meier method was used to analyze complication-free catheter survival. Statistical significance was set at $\mathrm{P}<0.05$.

\section{RESULTS}

The total number of patients included in the study was 371, of which 119 had an implantable port in the chest and
252 had an implantable port in the arm. The mean age was 58.6 years, and there were more women in the arm port group. The types of cancer included were colon cancer (157 patients; 42.3\%), breast cancer (156; 42.1\%), gastric cancer $(38 ; 10.2 \%)$, rectal cancer $(18 ; 4.9 \%)$, and pancreatic cancer $(2 ; 0.5 \%)$. The use of an arm port was most common in breast cancer patients, while chest ports were most commonly used in colon cancer patients $(P<0.001)$. A total of 142 patients with metastatic cancer and 290 patients who received adjuvant chemotherapy were included. Among them, the chest port was used more frequently for metastatic cancer ( $49.6 \%$ vs $32.9 \% ; \mathrm{P}=0.003$ ) and adjuvant chemotherapy ( $86.6 \%$ vs $74.2 \%$; $\mathrm{P}=0.011$ ) than the arm port. The demographics of the patients included in the study are summarized and described in detail in Table 1.

The success rate for both procedures was $100 \%$. All patients using the chest port had a catheter inserted through the right or left internal jugular vein, and the ports were mounted on the anterior chest wall. The most common veins accessed in the arm port group were the basilic vein in 204 patients (81\%), the cephalic vein in 35 patients (13.9\%) and the brachial vein in 13 patients $(5.1 \%)$. The

Table 1. Demographics and Baseline Characteristics

\begin{tabular}{|c|c|c|c|c|}
\hline Patient characteristics & Chest $(n=119)$ & $\operatorname{Arm}(\mathrm{n}=252)$ & Total $(\mathrm{n}=371)$ & $\mathrm{P}$ \\
\hline Age, year & $64.5 \pm 11.4$ & $55.8 \pm 11.4$ & $58.6 \pm 12.1$ & $<0.001$ \\
\hline Female, n (\%) & $52(43.7)$ & $172(68.3)$ & $224(60.4)$ & $<0.001$ \\
\hline Body mass index $\left(\mathrm{kg} / \mathrm{m}^{2}\right)$ & $23.2 \pm 3.5$ & $24.4 \pm 3.9$ & $24.0 \pm 3.8$ & 0.319 \\
\hline Weight (kg) & $59.5 \pm 11.2$ & $62.5 \pm 11.0$ & $61.6 \pm 11.2$ & 0.016 \\
\hline Height $(\mathrm{cm})$ & $160.1 \pm 8.6$ & $160.1 \pm 7.4$ & $160.1 \pm 7.8$ & 0.964 \\
\hline Smoking & $13(10.9)$ & $32(12.7)$ & $45(12.1)$ & 0.625 \\
\hline Hypertension & $49(41.2)$ & $78(31.0)$ & $127(34.2)$ & 0.053 \\
\hline Diabetes mellitus & $19(16.0)$ & 37 (14.7) & $56(15.1)$ & 0.747 \\
\hline Cerebrovascular disease & $2(1.7)$ & $6(2.3)$ & $8(2.1)$ & 0.122 \\
\hline Anti-Platelet agent & $6(5.0)$ & $5(2.0)$ & $11(3.0)$ & 0.186 \\
\hline Anti-Coagulants & $0(0.0)$ & $3(1.2)$ & $3(0.8)$ & 0.554 \\
\hline Cancer type & & & & $<0.001$ \\
\hline Breast & $15(12.6)$ & $141(56.0)$ & $156(42.1)$ & \\
\hline Colon & $88(74.0)$ & $69(27.4)$ & $157(42.3)$ & \\
\hline Rectum & $3(2.5)$ & $15(6.0)$ & $18(4.9)$ & \\
\hline Stomach & $13(10.9)$ & $25(9.9)$ & $38(10.2)$ & \\
\hline Pancreas & $0(0.0)$ & $2(0.8)$ & $2(0.5)$ & \\
\hline Metastasis & $59(49.6)$ & $83(32.9)$ & $142(38.3)$ & 0.003 \\
\hline Adjuvant & $103(86.6)$ & $187(74.2)$ & $290(78.2)$ & 0.011 \\
\hline Radiation therapy & $20(16.8)$ & $128(50.8)$ & $148(39.9)$ & $<0.001$ \\
\hline
\end{tabular}


mean length of the inserted conduit was 46.5, and the mean diameter of the veins was $3.1 \mathrm{~mm}$ (Table 2).

The most common complications were infection-related insults $(n=28,7.5 \%)$, and there was no statistically significant difference between the two groups in terms of events that could be deemed early complications, occurring within 2 weeks. There was no significant difference between the groups in terms of complications related to thrombosis ( $P=0.139$ ). Late complications (after 2 weeks), such as catheter-related blood stream infection (CRBSi), pocket infection, and wound dehiscence occurred more frequently in the chest port group than in the arm port group, and the difference was statistically significant $(\mathrm{P}=$ 0.031). Overall total complication rates were also lower in the arm port group than in the chest port group $(\mathrm{P}<0.001)$. However, there was no difference in the duration of port implantation between the two groups $(\mathrm{P}=0.456)$. The complication-free survival rate was higher in the arm port

Table 2. Detail Profile of Arm Port Procedures

\begin{tabular}{lccr}
\hline $\begin{array}{c}\text { Arm port } \\
(\mathrm{n}=252)\end{array}$ & $\begin{array}{c}\text { Right } \\
(\mathrm{n}=74,29.4 \%)\end{array}$ & $\begin{array}{c}\text { Left } \\
(\mathrm{n}=178,70.6 \%)\end{array}$ & $\mathrm{P}$ \\
\hline Access vein, $\mathrm{n}(\%)$ & & & \\
Basilic vein & $59(79.7)$ & $145(81.4)$ & $<0.001$ \\
Brachial vein & $1(1.4)$ & $12(6.8)$ & 0.006 \\
Cephalic vein & $14(18.9)$ & $21(11.8)$ & 0.326 \\
Length (cm) & $44.4 \pm 3.2$ & $47.5 \pm 3.5$ & $<0.001$ \\
Diameter (mm) & $3.2 \pm 0.8$ & $3.0 \pm 0.7$ & 0.120 \\
\hline
\end{tabular}

group than in the chest port group $(\mathrm{P}<0.001, \mathrm{Fig} .2)$. The chest port group had more cases of port removal due to complications than the arm port group $(\mathrm{P}=0.002$, Table 3$)$. In the univariate and multivariate comparative analyses for risk factors of complications, the use of a chest port was found to be independent risk factor with statistically significant $(P=0.002$, Table 4).

\section{DISCUSSION}

The use of TIVAPs is increasing because it can provide

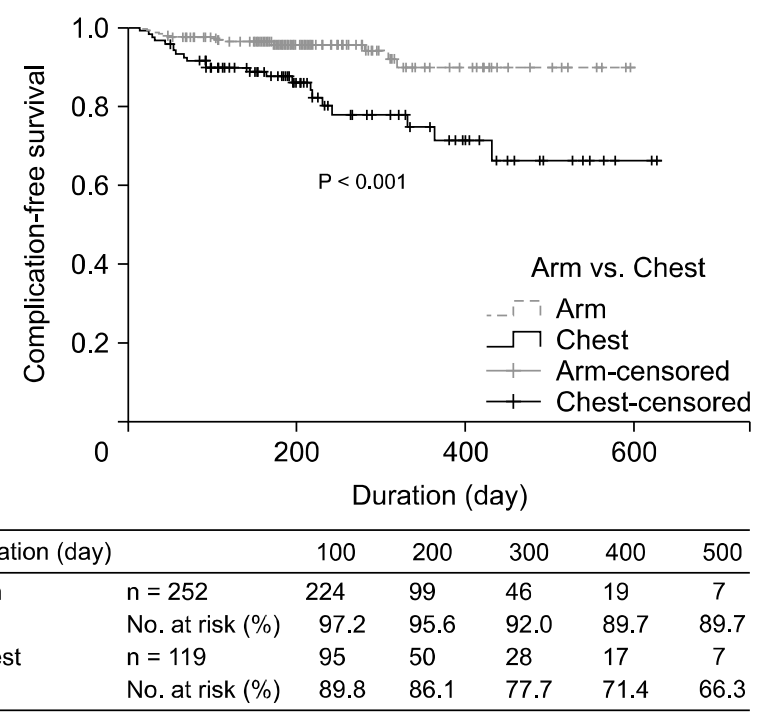

Fig. 2. Complication-free catheter survival.

Table 3. Comparison of Port-Related Complication in Arm and Chest Ports

\begin{tabular}{|c|c|c|c|c|}
\hline Follow-up & Chest $(\mathrm{n}=119)$ & $\operatorname{Arm}(\mathrm{n}=252)$ & Total $(\mathrm{n}=371)$ & $\mathrm{P}$ \\
\hline Thrombosis, n (\%) & $5(4.2)$ & $3(1.2)$ & $8(2.1)$ & 0.139 \\
\hline Early Infection, n (\%) & & & & $>0.999$ \\
\hline CRBSI & $1(50.0)$ & $1(100)$ & $2(66.7)$ & \\
\hline Pocket infection & $1(50.0)$ & 0 & $1(33.3)$ & \\
\hline Wound dehiscence & 0 & 0 & 0 & \\
\hline Late Infection, n (\%) & & & & 0.031 \\
\hline CRBSI & $2(12.5)$ & $6(66.7)$ & $8(32.0)$ & \\
\hline Pocket infection & $8(50.0)$ & $2(22.2)$ & $10(40.0)$ & \\
\hline Wound dehiscence & $6(37.5)$ & $1(11.1)$ & $7(28.0)$ & \\
\hline No. of complication $/ 1,000$ catheter day & $23(19.3)$ & $13(5.2)$ & $36(9.7)$ & $<0.001$ \\
\hline Catheter day/port (day) & $225.1 \pm 142.5$ & $214.1 \pm 109.8$ & $217.6 \pm 121.1$ & 0.456 \\
\hline Port removal, $\mathrm{n}(\%)$ & & & & 0.002 \\
\hline Finish of CTx & $72(60.5)$ & $155(61.5)$ & $227(61.2)$ & \\
\hline Complication & $20(16.8)$ & $12(4.8)$ & $32(8.6)$ & \\
\hline
\end{tabular}


Table 4. Univariate and Multivariate Analysis for Factors of Port-Related Complication

\begin{tabular}{|c|c|c|c|c|c|c|}
\hline \multirow{2}{*}{ Factor (reference) } & \multicolumn{3}{|c|}{ Univariate } & \multicolumn{3}{|c|}{ Multivariate } \\
\hline & HR & $95 \% \mathrm{CI}$ & $\mathrm{P}$ & HR & $95 \% \mathrm{CI}$ & $\mathrm{P}$ \\
\hline Sex (Female) & 1.265 & $0.650-2.462$ & 0.488 & 1.243 & $0.590-2.617$ & 0.567 \\
\hline Age (per year) & 0.989 & $0.962-1.017$ & 0.453 & 0.969 & $0.940-0.998$ & 0.040 \\
\hline BMI (per kg/m²) & 0.989 & $0.905-1.081$ & 0.813 & 1.000 & 0.911-1.098 & 0.997 \\
\hline Location of port (Arm) & 0.297 & $0.149-0.591$ & 0.005 & 0.240 & $0.115-0.503$ & 0.002 \\
\hline Smoking & 0.861 & $0.304-2.442$ & 0.778 & 0.986 & $0.324-3.003$ & 0.981 \\
\hline Hypertension & 0.575 & $0.268-1.23$ & 0.153 & & & \\
\hline Diabetes mellitus & 0.802 & $0.310-2.075$ & 0.649 & & & \\
\hline Anti-Coagulants & 3.018 & $0.411-22.179$ & 0.278 & & & \\
\hline \multicolumn{7}{|l|}{ Cancer type (Breast) } \\
\hline Colon and rectum & 1.265 & $0.613-2.612$ & 0.525 & & & \\
\hline Stomach & 0.720 & $0.201-2.579$ & 0.614 & & & \\
\hline Metastasis & 0.820 & $0.399-1.687$ & 0.591 & & & \\
\hline Neoadjuvant & 0.952 & $0.432-2.098$ & 0.902 & & & \\
\hline Radiation therapy & 0.766 & $0.365-1.608$ & 0.481 & & & \\
\hline
\end{tabular}

convenience and safety to patients who need frequent venous access. $(3,7)$. Especially, its safety has been proven for cancer patients who need chemotherapy, and the use of TIVAPs is recommended in guidelines provided by many cancer-related societies. $(2,3,7,8)$

The most used vein for the TIVAPs procedure is the internal jugular vein, and a port is inserted in the upper portion of the anterior chest wall. However, patients who have undergone breast cancer surgery or have head and neck cancer tend to pose challenged for insertion or reluctant to use these traditional methods. $(2,6,7)$ In such situations, an arm port can be an alternative method. Compared to the chest port, the arm port has no difference in the incidence of complications or safety and is a preferred method for breast cancer patients who value cosmesis and have aesthetic considerations. $(5,7)$

The most important aspect of performing the arm port procedure is the accurate puncture of an appropriate vein without damaging the surrounding structures. As such, it is recommended to use the Seldinger technique under ultrasound guidance. As shown in a randomized controlled study, compared to the establishment of access through a cutdown procedure, the Seldinger technique is a much more effective and faster method for venous access and is highly recommended for most patients who require venous port access.(9)
The location of the catheter tip is also an important factor in determining the outcome of the port function, and it is generally important to position the catheter tip at the confluence point of the right atrium and the superior vena cava. Therefore, radiologic confirmation must be achieved. $(5,10,11)$

The position in which the port is inserted into the patient's arm may also be an important factor in terms of patient convenience. If it is located close to the basilic vein, it is inconvenient because the patient needs to rotate the arm internally and the needling site may be disrupted, which may cause complications.(7) In addition, if the basilic vein of the upper arm is punctured and the port is placed in the forearm, there may be limitations in arm movement, and the catheter may be damaged by continuous motion of the limb. $(7,12)$ For this reason, upon puncture of the basilic or brachial veins of the upper arm in our hospital, the port is placed on the lateral side of the upper arm; meanwhile, when the cephalic vein is punctured, the port is inserted near the puncture site.

For veins of the upper arm to be punctured, it is generally recommended to use veins with a diameter of $3 \mathrm{~mm}$ or more. $(8,10)$ We used basilic or cephalic veins with a diameter of more than $3 \mathrm{~mm}$ whenever possible; however, brachial veins were used when the diameter the other veins was less than $2 \mathrm{~mm}$ or if pathologies otherwise precluded 
the use of the superficial vein. In some cases, appropriate venous access was possible even with a diameter of $3 \mathrm{~mm}$ or less through careful, ultrasound-guidance. If vein access was still not possible, a cut-down procedure was performed through a $1 \mathrm{~cm}$ mini-incision, allowing for a technical success of $100 \%$.

Although TIVAPs-related complications vary between several studies, they are reported at 0.3-1.8/1000 catheter days, and the most common complications are thrombosis and infection.(6) A systemic review and retrospective study have shown that TIVAPs placements between the chest and the arm did not have significant difference in terms of adverse events for breast cancer patients. Moreover, patient satisfaction for arm port placement have shown better results than for chest port placement. $(2,6,10)$ We conducted this study to determine whether TIVAPs port placement on the arm would be applicable to other cancer patients as well as breast cancer.

Thrombosis can be very serious and affect the prognosis of cancer patients. These events are closely related to an increased risk of venous thromboembolism among cancer patients.(3) Unfortunately, there is no prophylaxis to prevent catheter-related VTE among cancer patients, and anticoagulant administration is recommended for high-risk patients with VTE. $(8,13)$ In our study, there was no statistically significant difference in the incidence of thrombosis between the arm and chest port groups.

Catheter-related infections are generally reported in $5.3 \%-13.0 \%$ of cases and are also an important variable in determining the prognosis of cancer patients. (5) Previous studies reported that the arm port had a higher incidence of infection than the chest port, whereas our study showed the opposite result.(2) As the results show, there was no difference in the incidence of thrombosis or catheter-related complications between the two groups; however, incision-related complications occurred more frequently in the chest port group. Even though the chest port group had a smaller number of patients, the fact that there were more complications related to wounds in the chest port group seems to have many implications. Considering the reason, the port to be inserted into the arm is $6 \mathrm{~F}$, and the port to be inserted into the chest is $8 \mathrm{~F}$, which is larger in terms of diameter and thickness. According to the access venous size, the incision windows tend to be larger with increasing caliber and thickness of the access port for insertion. In addition, the distance between the port and the skin is closer to in chest group because the subcutaneous fat layer in the chest is thinner than the same layer in the arm, and the pressure on the skin due to protruding ports can also be higher in the chest port insertion group. It is possible that incision-related complications more frequently occurred in the chest port group for these reasons. However, previous studies have shown no difference in the incidence of infection complications between the two groups. $(6,7)$ It should be considered that bias in this study may influence its results like differences of cancer type, sex, and age. In any consideration, since successful establishment for port implantation is an important aspect of management in the administration of chemotherapy and can affect the schedule of antineoplastics in the event of complications, particular attention should be paid to wound sutures.

Finally, there are few studies on the use of intravenous access ports for patients with metastatic cancer. Lebeaux et al.(14) reported that the incidence of catheter-related infections was high in patients with metastasis or palliative treatment. In addition, there are few studies comparing complications between arm and chest venous access ports among metastatic cancer patients; in our study, location for access port placement was not associated with any difference in incidence of complications among metastatic cancer patients.

This study has two important limitations. First, this is a retrospective study, and so patient selection bias may present. Second, heterogeneity between the two groups of patients could have affected the results of the study.

\section{CONCLUSION}

TIVAPs is a very important procedure for patients who need chemotherapy. With the risk of complications such as 
thrombosis and infection, it is necessary to consider and select an appropriate location where venous access can be established prior to the procedure. In this study, it was found that the arm port group had a lower complication rate than the chest port group, however, a further reviews and studies on this issue are needed.

\section{ETHICS APPROVAL}

The work was carried out in accordance with the Code of Ethics of the World Medical Association (Helsinki Declaration) for experiments on humans. This study was approved by the Institutional Review Board of Jeonbuk National University Hospital (CUH 2020-02-028-003).

\section{CONFLICT OF INTEREST}

All authors declare that they have no conflict of interest.

\section{FUNDING}

This Article was supported by Fund of Research Institute of Clinical Medicine of Jeonbuk National University and Biomedical Research Institute of Jeonbuk National University Hospital.

\section{AUTHORS’ CONTRIBUTIONS}

Conceptualization: HPH. Data curation: BHC. Formal analysis: JDY. Methodology: BHC. Project administration: HCY. Visualization: MRL. Writing - original draft: JDY, Writing - review \& editing: HPH and HCY.

\section{ACKNOWLEDGEMENTS}

The authors thank the Research Institute of Clinical Medicine of Jeonbuk National University-Biomedical Research Institute of Jeonbuk National University Hospital -for supporting this study through grant funding and access to experimental facilities.

\section{REFERENCES}

1. Kato K, Iwasaki Y, Onodera K, Higuchi M, Kato K, Kato Y, et al. Totally implantable venous access port via the femoral vein in a femoral port position with CT-venography. J Surg Oncol 2016;114:1024-8.

2. Li G, Zhang Y, Ma H, Zheng J. Arm port vs chest port: a systematic review and meta-analysis. Cancer Manag Res 2019;11: 6099-112.

3. Gallieni M, Pittiruti M, Biffi R. Vascular access in oncology patients. CA Cancer J Clin 2008;58:323-46.

4. Sousa B, Furlanetto J, Hutka M, Gouveia P, Wuerstlein R, Mariz JM, et al. Central venous access in oncology: ESMO clinical practice guidelines. Ann Oncol 2015;26 Suppl 5:v152-68.

5. Yang SS, Ahn MS. A comparison between upper arm and chest for optimal site of totally implanted venous access ports in patients with female breast cancer. Ann Vasc Surg 2018;50: 128-34.

6. Xu H, Chen R, Jiang C, You S, Zhu Q, Li Y, et al. Implanting totally implantable venous access ports in the upper arm is feasible and safe for patients with early breast cancer. J Vasc Access 2020;21:609-14.

7. Marcy PY, Figl A, Amoretti N, Ianessi A. Arm port implantation in cancer patients. Int J Clin Oncol 2010;15:328-30.

8. Magallón-Pedrera I, Pérez-Altozano J, Virizuela Echaburu JA, Beato-Zambrano C, Borrega-García P, de la Torre-Montero JC. ECO-SEOM-SEEO safety recommendations guideline for cancer patients receiving intravenous therapy. Clin Transl Oncol 2020;22:2049-60.

9. Nocito A, Wildi S, Rufibach K, Clavien PA, Weber M. Randomized clinical trial comparing venous cutdown with the Seldinger technique for placement of implantable venous access ports. Br J Surg 2009;96:1129-34.

10. Bertoglio S, Cafiero F, Meszaros P, Varaldo E, Blondeaux E, Molinelli C, et al. PICC-PORT totally implantable vascular access device in breast cancer patients undergoing chemotherapy. J Vasc Access 2020;21:460-6.

11. Marcy PY, Magné N, Castadot P, Italiano A, Amoretti N, Bailet $\mathrm{C}$, et al. Is radiologic placement of an arm port mandatory in oncology patients? analysis of a large bi-institutional experience. Cancer 2007;110:2331-8.

12. Kehagias E, Tsetis D. The "Arm-to-Chest Tunneling" technique: a modified technique for arm placement of implantable ports or central catheters. J Vasc Access 2019;20:771-7.

13. Practice Guidelines for Central Venous Access 2020: an updated report by the American Society of Anesthesiologists Task Force on Central Venous Access. Anesthesiology 2020;132:8-43.

14. Lebeaux D, Larroque B, Gellen-Dautremer J, Leflon-Guibout V, Dreyer C, Bialek S, et al. Clinical outcome after a totally implantable venous access port-related infection in cancer patients: a prospective study and review of the literature. Medicine (Baltimore) 2012;91:309-18. 